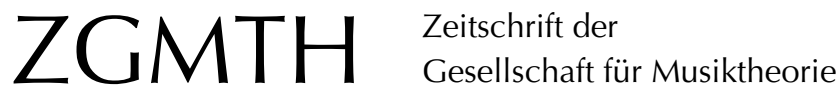

Polth, Michael (2006): Tonalität der Tonfelder. Anmerkungen zu Bernhard Haas (2004), Die neue Tonalität von Schubert bis Webern. Hören und Analysieren nach Albert Simon, Wilhelmshaven: Noetzel. ZGMTH 3/1, 167-178.

https://doi.org/10.31751/210

(C) 2006 Michael Polth

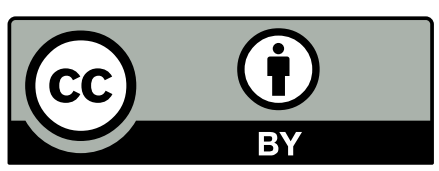

Dieser Text erscheint im Open Access und ist lizenziert unter einer Creative Commons Namensnennung 4.0 International Lizenz.

This is an open access article licensed under a

Creative Commons Attribution 4.0 International License.

veröffentlicht / first published: 01/01/2006

zuletzt geändert / last updated: 14/12/2008 


\section{Tonalität der Tonfelder. Anmerkungen zu Bernhard Haas, Die neue Tonalität von Schubert bis Webern. Hören und Analysieren nach Albert Simon, Wilhelmshaven: Noetzel 2004}

Die historiographische Vorstellung, die sich vom Übergang der sogenannten 'Dur-MollTonalität in die sAtonalität im allgemeinen Bewusstsein festgesetzt hat, ist diejenige von Arnold Schönberg. Nach Schönberg hatte die Tonalität im 19. Jahrhundert durch die Verwendung sentfernter Stufen<, durch die Anreicherung der Akkorde mit chromatischen Tönen, durch eine freiere Dissonanzbehandlung sowie durch den Einsatz svagierender Akkorde، an form- und zusammenhangsbildender Kraft verloren (nach Dahlhaus ging die Aufgabe der Zusammenhangsbildung an die motivischthematische Arbeit über). Der Übergang in die sAtonalitätı, der zugleich eine Emanzipation der Dissonanzen aus ihrer satztechnischen Abhängigkeit von konsonanten Intervallen und eine Emanzipation der Zusammenklänge aus der Hierarchie der Tonart darstellen sollte, war somit kein Bruch mit der traditionellen Tonalität, sondern deren konsequente Weiterentwicklung. Bei aller Sympathie für eine Sichtweise, in der die >Atonalität von der `DurMoll-Tonalität nicht durch einen Systemwechsel getrennt ist (nach der sich also die Musik vor 1911 bereits von einer Tonika ebenso emanzipiert hat, wie die Musik danach immer noch einen harmonischen Zusammenhang konstituiert), fällt es aufgrund der Tatsache, dass alle Theorien der Tonalität auf dem Tonikabezug als Grundprinzip beruhen, schwer, die Kontinuität in der Geschichte der Tonalität um das Jahr 1911 zu begreifen. Vorzugsweise bildet der Beginn der Atonalität im allgemeinen Bewusstsein eine Zäsur. Und die Kehrseite der übertriebenen Vorstellung von der Zäsur um 1911 ist die übertriebene Vorstellung von der Einheit der Jahre 1600 bis 1911 als eines Zeitraums der einen sDur-Moll-Tonalitätı.

Eine Hilfe könnte das Buch Die neue Tonalität von Schubert bis Webern. Hören und Analysieren nach Albert Simon von Bernhard Haas bieten. Das Buch skizziert eine Tonalität, die sich nicht durch Tonikabezug, sondern durch die Bildung von Tonfeldern konstituiert. Historisch gesehen fällt die ‘klassische Zeit dieser Tonalität in die zweite Hälfte des 19. und die erste des 20. Jahrhunderts. Die charakteristischen harmonischen Zusammenhänge und Effekte, auf welche die Theorie der Tonfelder vorzugsweise zielt, sind solche, wie man sie vor allem aus dem späten 19. Jahrhundert kennt. Der erste Komponist, in dessen Werken diese Zusammenhänge und Effekte mit einer solchen Deutlichkeit auftreten, dass sie eine Analyse durch Tonfelder geradezu herausfordern, ist allerdings Franz Schubert gewesen. Einzelne Effekte finden sich sogar schon seit dem `Zerfall der barocken Musikanschauung، von etwa 1730 an, z. B. bei C. Ph. E. Bach. Das Augenmerk des Buches fällt aber nicht nur auf die Musik aus der zweiten Hälfte des 19. Jahrhunderts, sondern auch auf Kompositionen nach 1911, darunter dodekaphone Werke; denn nach der These des Buches konstituiert sich auch noch in den zwölftontechnischen Kompositionen Schönbergs, Bergs und Weberns der musikalische Zusammenhang durch Tonfelder (die Zwölftontechnik selbst ist kein Modus der Zusammenhangsbildung). 


\section{Grundthesen}

Bei der Gesamtperspektive, die das Buch bietet, ist sorgfältig zwischen den Anteilen von Albert Simon und Bernhard Haas zu unterscheiden. Auf Albert Simon, einen im Jahre 2000 verstorbenen ungarischen Dirigenten, geht die Theorie der Neuen Tonalität im ganzen zurück. Von dieser Theorie existiert ein vollendetes Manuskript, das momentan nicht zur Veröffentlichung freigegeben ist. Alle Beschreibungen der Tonfelder, die Bernhard Haas (der Simon persönlich gekannt hat) anbietet, referieren lediglich die Überlegungen Simons. Die Analysen einzelner Werke von Schubert, Alkan, Franck, Liszt, Schönberg und Webern stammen allerdings von Haas selbst. Und über Simon hinaus geht Haas durch die Installation einer historischen Perspektive, also durch die Einordnung der Tonfeld-Tonalität in eine Geschichte der Tonalitäten überhaupt (wobei zu bemerken wäre, dass Simon selbst nie verkannt hat, welchen historischen Ort die von ihm beschriebene Tonalität innehat).

Bernhard Haas ist ein ausgesprochener Kenner der Schenkerschen Theorie und Analyse. Aus seiner Sicht heraus hat in der Zeit zwischen 1730 und 1850 eine allmähliche Ablösung der >Ursatz-Tonalitätı nach Schenker durch eine `Tonfeld-Tonalität n nach Simon stattgefunden. Innerhalb der >durmolltonalen Epocher hat es somit einen Systemwechsel gegeben. Wer einerseits bei Händel und andererseits bei Liszt von einer Komposition in C-Dur spricht, meint - bewusst oder unbewusst - zwei in ihrem grundsätzlichen Funktionieren völlig verschiedene Tonarten.

Die spannenden Fragen, die die Ausführungen von Haas aufwerfen, betreffen die beiden ,Übergangszeiten : 1730 bis 1850 und 1890 bis 1920 . Haas hatte sich nicht zum Ziel gesetzt, über die Darstellung der Theorie Simons hinaus auch diese problematischen Zeit- spannen eingehend zu behandeln. Aber das Buch bietet für beide Zeiträume Perspektiven. So lassen sich nach Haas die Kompositionen Haydns, Mozarts, Beethovens, Schuberts, Schumanns, Chopins und Mendelssohns sowohl nach Schenker als auch nach Simon erfolgreich analysieren. Anhand der Analyse eines Liedes von Schubert gibt er die Richtung vor, in die ein Versuch zu gehen hätte, der das Zusammenbestehen zweier Analysemethoden bei einer Komposition in eine autonome Beschreibung des Zustandes der Tonalität in diesem Werk überführen möchte. Die (durchaus vielversprechende) Hypothese lautet, dass die harmonischen Zusammenhänge und Effekte, auf die die Tonfelder zielen, zunächst in Fantasien, Durchführungen und kurzen Abschnitten als Sondererscheinungen auftauchen, während die Komposition im ganzen durch die Funktionen der Schenkerschen Theorie zusammengehalten wird. Der Prozess bis zur Mitte des 19. Jahrhunderts bestünde darin, dass die Tonfeld-Effekte nicht nur selbstverständlicher werden, sondern die Funktionen Schenkers in ihrer formbildenden Rolle ablösen.

Genauer gesagt: Schenkers Analysen zeigen, wie Kompositionen in ihrem Verlauf Strukturen vollenden oder einlösen (solche Strukturen sind Züge, Brechungen, Ausfaltungen, Koppelungen usw.). Immer klärt das spätere Geschehen über das frühere auf, indem es im Nachhinein deutlich macht, wie das frühere Teil einer Struktur gewesen ist. Die harmonischen Effekte, die von Schenkers Blick bevorzugt getroffen werden, sind solche, die entweder aus dem Einlösen einer Struktur oder aus dem Verzögern ihrer Vollendung resultieren (so beispielsweise der charakteristische Klang einer Subdominante, wenn sie als ,Prolongation den Eintritt der Dominante verzögert). Wenn also die Funktionen Schenkers durch die Tonfelder Simons abgelöst wurden, 
dann wurde damit auch die finale Ausrichtung der Kompositionen, die Orientierung am Späteren (das über den Sinn des Früheren aufklärt) preisgegeben. Mit dieser Orientierung verschwanden bestimmte harmonische Effekte aus der Musik. Es reicht ein kurzer Blick auf die Musik des späten Debussy, um zu sehen, dass es hier den Effekt einer lange vorbereiteten und ssatten s Schlusstonika oder einen stringenten Gang des Basses nicht mehr gibt. Umgekehrt ist das für Debussy charakteristische ।In-sich-Bewegen von stehenden Klangflächen einer der Effekte, auf den die Theorie der Tonfelder zielt.

Auch für die zweite Übergangszeit von 1890 bis 1920 bietet Haas eine Perspektive an. Wie Schönberg richtig gesehen hat, ist die Preisgabe der Tonika ein - systematisch gesehen - kleiner Schritt in der Entwicklung der Tonalität gewesen. Die tiefgreifenden Veränderungen der Tonalität hatten bereits ein halbes Jahrhundert vor der sAtonalitäts stattgefunden, als - nach Simon/Haas - die Tonalität der Tonfelder zur Systemgrundlage des musikalischen Zusammenhangs geworden war. Im Gegensatz zur alten Tonalität (nach Schenker), in der ein Tonartbezug von substantieller Bedeutung ist, weil sich der musikalische Zusammenhang durch die Darstellung einer Tonika konstituiert, ist die Darstellung einer Tonika in einer Musik, die auf Tonfeldern beruht, sekundär. Die Akkorde geben sich nicht dadurch einen Zusammenhang, dass sie eine bestimmte Tonart begründen, sondern zu denselben oder verschiedenen Tonfeldern gehören. So hört man die Emanzipation des harmonischen Zusammenhangs von einer Tonart beispielsweise daran, dass die Tonart h-Moll in der h-Moll-Sonate von Liszt nur noch an formalen Eckpunkten präsent ist. Der überwiegende Teil der Akkordfortschreitungen lässt sich von einem Bezug zu h-Moll oder überhaupt zu einer bestimmten Tonart gar nicht oder nur unzureichend verstehen.

Die Hauptstrukturen einer Tonart bei Schenker führen notwendigerweise immer bis in den strukturellen Schluss einer Komposition (der nicht mit dem realen Schluss zusammenfallen muss), die beiden großen Tonfelder, die nach Haas eine Komposition bestimmen (auf der obersten Ebene einer Komposition spielen immer zwei Tonfelder eine Rolle), müssen mit dem Schluss einer Komposition nicht zwingend etwas zu tun haben. Man kann hierbei zum Beispiel an die Symphonische Fantasie und Fuge von Max Reger denken, deren Schlusskadenzen nicht mehr (im Sinne Schenkers) über das (dichtgedrängte und turbulente) harmonische Geschehen zuvor aufklären.

Die Geschichte vom Wandel der Tonalität, die das Buch von Haas skizziert, ist im großen und ganzen gesehen vielleicht nicht neu oder überraschend. Dass in der Musik Debussys Klangflächen eine Rolle spielen, hat man durchaus schon gewusst. Aber durch die Fundierung der Historiographie in den Theorien von Schenker und Simon gewinnt die Skizze an Tiefe, weil sich die Behauptungen bis in die konkreten Einzelheiten der Tonsätze hinein verfolgen lassen.

\section{Zur Logik der Analyse}

Simons Theorie hat unverkennbare Ähnlichkeiten mit Theoremen von Lendvai ${ }^{1}$ und Gardonyi ${ }^{2}$. Zahlreiche Einzelheiten finden sich bereits als Beobachtungen in Richard Taruskins Strawinskymonographie. ${ }^{3}$ Darüber hinaus berührt sich das Anliegen Simons mit dem der `Neo-Riemannian Theory eines Richard Cohn. ${ }^{4}$

Über Lendvai, Gardonyi und Taruskin geht Simon allerdings durch die Fülle der Beobach- 
tungen und deren systematische Durchdringung hinaus. Mit der gebotenen Vorsicht darf man sagen, dass es Simon innerhalb der Tonalität der zweiten Hälfte des 19. und der ersten Hälfte des 20. Jahrhunderts gelungen ist, zahllose Erscheinungsformen zu sammeln, systematisch zu ordnen und auf grundlegende Prinzipien zu bringen. Und man darf einem Autor, der sich in lebenslanger Beschäftigung einen theoretischen Zugang erarbeitet hat, welcher musikalische Erscheinungen eines bestimmten Zeitraums beinahe sflächendeckend erfasst, ohne dass der umfassende Blick durch leere Abstraktion erkauft würde, zutrauen, dass er etwas hinsichtlich der grundsätzlichen Verfasstheit der von ihm behandelten Musik verstanden hat (dies um so mehr, als Simon - wie gesagt - nicht grundsätzlich Neues behauptet, sondern vorzugsweise Einzelbeobachtungen seiner Vorgänger in einen größeren Rahmen gestellt hat).

Jede Musiktheorie, die es mit einer systematisch erschlossenen Fülle von Erscheinungen zu tun hat, folgt einer anderen Logik als Fallstudien. Wie Schenkers Theorie ist auch diejenige von Simon weder deskriptiv noch normativ, sondern - wenn überhaupt korrektiv. Sie beschreibt nicht, was der Fall ist (das wäre entweder belanglos, wenn es sich um überprüfbare Äußerlichkeiten handelte, oder unmöglich, wenn es sich um Gegenstände des musikalischen Zusammenhangs handelt, die sich in einem Subjekt konstituieren). Aber sie schreibt der Musik auch nicht a priori vor, wie ihr Zusammenhang beschaffen ist (das wäre der Fall, wenn sie aus Gründen, die unabhängig von den Kompositionen bestehen, Regeln über die Bildung von musikalischem Zusammenhang herleiten würde). Das Ziel der Theorien von Schenker und Simon ist es vielmehr, das Hören durch eine Fülle bereitgestellter Begriffe, die auf Klangwirkungen zielen, zu differenzieren. ${ }^{5}$ Das Interesse gilt weder der vermeintlichen Beschaffenheit von Kompositionen (als Faktum an sich) noch der empirischen Vielfalt der Wahrnehmung von Hörern, sondern der Kommunikation zwischen Hörer und Gegenstand. Die Theorie sagt nicht, wie der Gegenstand beschaffen ist, und beschreibt nicht, wie gehört wird, sondern möchte Einfluss auf die Wahrnehmung der Musik nehmen. Sie sinnt den Leser an, das erklingende Kunstwerk auf eine bestimmte Weise wahrzunehmen. Und sie sbeweist ihre Triftigkeit alleine dadurch, dass die Differenzierungen, die sie anbietet, dem Hörer tatsächlich zu einer neuen detaillierten Wahrnehmung verhelfen, in der sich ihm die Fülle und der Reichtum eines musikalischen Kunstwerks erschließen.

Zur Logik dieser Theorien gehört, dass nach der Realität der Differenzierungen nicht naiv gefragt werden kann - in der Art, ob die Unterschiede, die sich durch eine Beschäftigung mit Schenker oder Simon hören lassen, wirklich existieren oder ob sie nur eingebildet sind. Eine Antwort darauf gäbe vor zu wissen, wie das Kunstwerk oder der Hörer seigentlich، beschaffen sei. Wenn jedoch die Differenzierungen, die Schenker und Simon ihren Lesern ansinnen, nur in der Relation zwischen dem Hörer und dem Kunstwerk zustande kommen, wenn sich Tonalität überhaupt nur in einem Akt der Kommunikation konstituiert ${ }^{6}$, dann ist das Erlebnis einer differenzierten Wahrnehmung, die mit Bestimmtheit erfahren wird, bereits die Realität, um die es geht. Einen Standpunkt, von dem aus entschieden werden könnte, ob die erlebte Differenzierung san sich richtig ist, gibt es nicht. - Auf die Bestimmtheit des ästhetischen Erlebnisses kommt es an, und diese lässt sich wiederum daran erfahren, dass derselbe Modus der Wahrnehmung an anderen Stellen der Komposition oder an anderen Kompositionen versagt. Die Form einer Wahrnehmung lässt sich 
einem Kunstwerk nicht beliebig überstülpen. Oder aber: Wer nie scheitert, hat etwas falsch gemacht. (Dabei muss sich Bestimmtheit nicht beim ersten Versuch einstellen, sie kann das Ziel einer längeren Beschäftigung sein.)

\section{Arten der Tonfelder}

Simon kennt drei Arten von Tonfeldern: ^Funktion`, 'Konstrukt ‘ und `Quintenreihe`. Jedes ist nach einer eigenen Gesetzmäßigkeit gebaut.

Die 'Funktion` besteht aus vier Grundtönen im Kleinterzabstand sowie aus den vier Quinttönen der Grundtöne (Beispiel 1). Die acht Töne entsprechen der Halbton-GanztonSkala (`Oktotonik`) oder dem zweiten Modus von Messiaen. Es gibt drei Funktionen (beginnend mit $c$, cis und $d$; die Funktion auf es entspricht der ersten).

Das «Konstrukt ‘ besteht aus drei Tönen im Großterzabstand sowie deren Quinttönen (Beispiel 2). Die sechs Töne bilden eine Skala aus Halbtonschritten und Kleinterzen. Es gibt vier Konstrukte.

Die 'Quintenreihe besteht aus drei bis zehn Tönen im Abstand von reinen Quinten (Beispiel 3). Nach der Zahl der Töne wird unterschieden zwischen `Triton`, `Tetraton`, ,Pentaton<, 'Hexaton usw.

Tonfelder sollen helfen, bestimmte Klangwirkungen, die vor allem die Harmonik von 1850 an prägen, differenzierter zu verstehen. Drei wesentliche Eigenarten der sspätromantischen< Tonalität sind bereits durch die Eigenschaften der Tonfelder impliziert:

- dass der Akkord-Zusammenhang nicht durch eine Tonart vermittelt werden muss,

- dass zusammenhängende Töne nicht immer eine klare Diskretion in bestimmte Akkorde (im traditionellen Sinne) zulassen und

- dass Akkorde, die in ihrer Klangwirkung voneinander abhängen, satztechnisch nicht auf gleicher Ebene liegen müssen.
Beispiel 1: `Funktion` nach Simon

Beispiel 2: «onstruktı nach Simon

Beispiel 3: ^Quintenreihe nach Simon

Beispiel 4: Franz Liszt, Orpheus, stonikale Funktions des Beginns
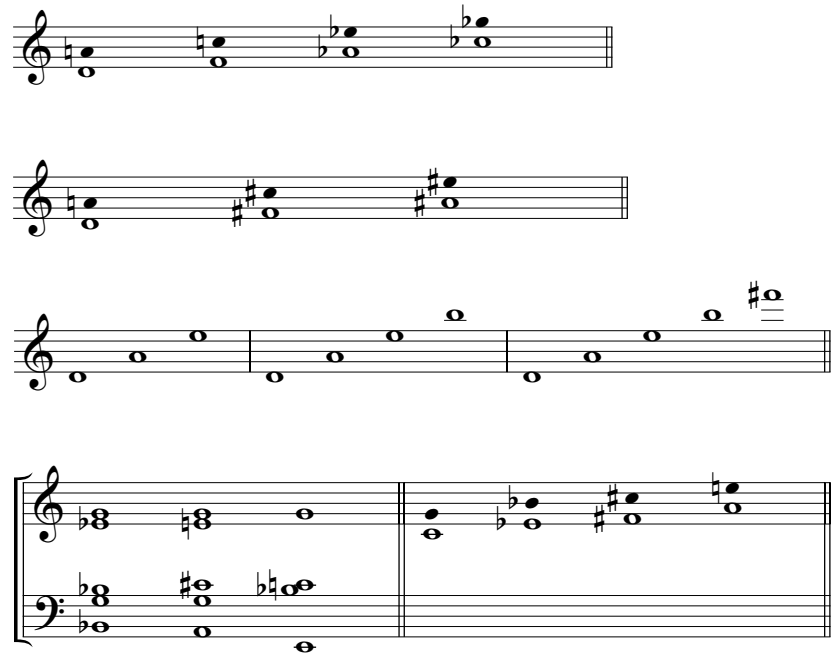
Vermittlung durch eine Tonart

Die Sinfonische Dichtung Orpheus von Liszt beginnt mit der Akkordfolge Es-Dur - A-DurSeptakkord-C-Dur. Obwohl man mit gewissem Recht behaupten darf, dass die Komposition insgesamt in C-Dur steht, bilden die drei Akkorde des Anfangs keinen Zusammenhang, der durch eine Tonart vermittelt wäre: Es-Dur muss nicht innerhalb von C-Dur als tP und A-Dur als $\mathrm{Tp}^{7}$ gehört werden (oder EsDur als T und A-Dur als (TP) [Tp]). Vielmehr erscheinen die Akkorde zunächst unmittelbar und schließlich durch ein Tonfeld aufeinander bezogen.

Die harmonische Wirkung eines jeden Akkordes resultiert zunächst daraus, welchen anderen Akkorden er folgt. A-Dur erfährt eine besondere Beleuchtung durch seine Stellung nach Es-Dur, C-Dur wiederum durch seine Position nach Es-Dur und A-Dur. Auch der gemeinsame Ton $g$, den die Hörner einstreuen, und die Bewegung des Basses zu Beginn $(b-a)$ prägen die Klangwirkungen.

Für die eigentümliche harmonische Wirkung der Akkorde haben die traditionellen Harmonielehren keinen Begriff. Er wäre mit Äquivalenzwirkung am besten beschrieben. Äquivalenz meint den Eindruck, dass verschiedene Akkorde wie unterschiedliche Seiten ein und desselben Klanges wirken. So ist der A-Dur-Akkord zwar neu und von Es-Dur unterschieden, und doch scheint er nur dasselbe aus einem anderen Blickwinkel zu sagen, was auch der Es-Dur-Akkord ausdrückt (die Äquivalenz von Akkorden ist nicht mit dem Stellvertretergedanken der Funktionstheorie zu verwechseln, bei dem Nebenakkorde den Platz einer Hauptfunktion einnehmen können). Die Äquivalenz ist eine der Wirkungen, die dem Hörer durch das Tonfeld 'Funktion` bewusst gemacht werden sollen. Nach Simon bilden die Akkorde Es-Dur, A-Dur-Septakkord und C-Dur verschiedene Ausschnitte aus derselben Funktion. Die harmonischen Beziehungen der Akkorde sind dadurch geprägt, dass sich ihre Töne sukzessiv zu einem Tonfeld ergänzen.

\section{Diskretion}

Die traditionellen Harmonielehren und die Theorie Schenkers gehen davon aus, dass jeder Ton des Tonsatzes danach bestimmt werden kann, ob er einem Akkord angehört oder einen Nebenton darstellt, ob er an der Konstituierung eines harmonischen Fundaments beteiligt ist oder nicht. Diese Prämisse, nach der Töne eine klare harmonische Diskretion zum Ausdruck bringen müssen, ist dem 18. und den ersten Jahrzehnten des 19. Jahrhunderts weitgehend angemessen. In der zweiten Hälfte des 19. Jahrhunderts jedoch, also in der Musik, auf welche die Theorie der Tonfelder zielt, häufen sich die Fälle, in denen zusammenhängende Töne keine klare Unterscheidung im skizzierten Sinne zu erkennen geben.

Die Begleitfigur, mit der Gabriel Fauré sein Impromptu op. 34 eröffnet, drückt keine klare Vorstellung davon aus, ob es sich um zwei verschiedene Akkorde handelt (As-Dur und bMoll) oder um einen As-Dur-Akkord mit drei Nebentönen. Die Vereinigung der Töne zu einem in sich bewegten Gesamtklang ist allerdings ästhetische Absicht. Denn wenn man versucht, die Sechzehntel in die eine oder andere Richtung zu svereindeutigen (also entweder einen ersten von einem zweiten Akkord oder harmonieeigene von harmoniefremden Tönen zu unterscheiden), verliert der Anfang seinen eigentümlichen Reiz.

Nach Simon lassen sich die sechs Töne als Tonfeld beschreiben, als sechstönige Quintenreihe (als Hexaton des-as-es-b-f-c), die 


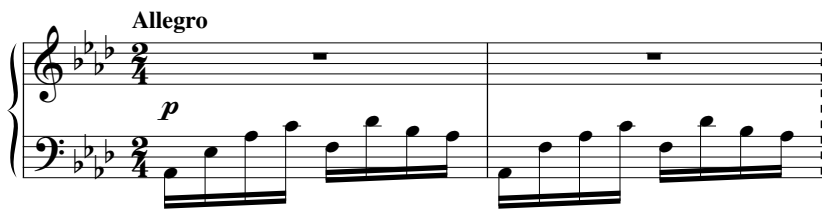

Beispiel 5: Gabriel Fauré, Impromptu op. 34, Beginn

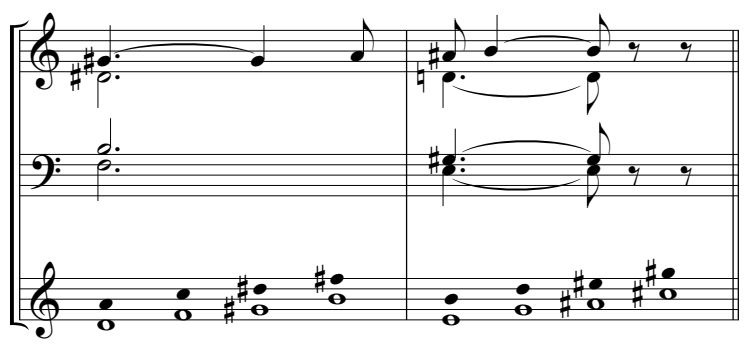

Beispiel 6: Wagner, Tristan-Vorspiel, Beginn nach ssubdominantischer und sdominantischer Funktion geordnet

sukzessiv in Bewegung gebracht wird (und zwar so, dass die Folge sowohl einen As-Durals auch einen b-Moll-Akkord andeutet). Mit dieser Beschreibung wird der Klangeindruck und damit der ästhetische Sinn der Stelle getroffen.

Ein berühmtes Beispiel für eine unbestimmte Diskretion ist der Tristan-Anfang. Bekanntlich wurde darum gestritten, ob der erste Akkord ein gis oder ein a enthält. Ist das gis Vorhalt zu a oder das a Durchgang zu ais? Die Unbestimmtheit ist kein Defizit, sondern gehört zum Wesen der Tristan-Harmonik. Simon kann dieser Unbestimmtheit gerecht werden, indem er eine Folge von zwei Funktionen annimmt. Die Töne gis und a gehören demnach beide zum ersten Tonfeld wie die
Töne ais und $h$ zum zweiten. Der traditionelle Unterschied zwischen harmonieeigen und harmoniefremd, der sich ästhetisch ohnehin nicht mehr mitteilt, ist in der Theorie der Tonfelder aufgehoben. Andererseits wird der Unterschied zwischen den Tönen gis und a nicht völlig nivelliert, sondern lediglich auf eine andere Ebene verlagert: gis und a heben sich nicht mehr als harmonieeigen und harmoniefremd voneinander $a b$, sondern als Grundton (gis) und Quintton (a) des Tonfeldes.

Nebenbei bemerkt: Das Beispiel zeigt, dass die Funktionen Simons unverkennbar an die Akkordfunktionen der Funktionstheorie anschließen. Sie gehen lediglich an Vollständigkeit und systematischer Ausweitung über diese hinaus. Denn die traditionellen Erklä-

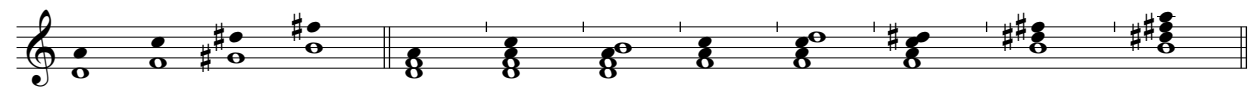

Beispiel 7: Wagner, Tristan-Vorspiel, ssubdominantische Funktion` und mögliche Ausschnitte des Tonfelds 
rungen, es handele sich beim Tristan-Anfang um eine Art von sphrygischem Halbschluss $d$ Moll - E-Dur) oder um einen Halbschluss mit der Funktionsfolge DD - D (H-Dur - E-DurSeptakkord), werden durch Simon prinzipiell nicht in Frage gestellt. Nur die Möglichkeit, dass sich der erste Akkord klar als subdominantischer oder doppeldominantischer Klang muss deuten lassen können, wird zurückgewiesen. Tatsächlich enthält das erste Tonfeld alle Töne, aus denen sich sowohl die ssubdominantischen als auch die sdoppeldominantischen Akkorde in a-Moll herausschneiden lassen: d-Moll, d-Moll-Septakkord, d-Moll mit ssixte ajoutée`, F-Dur, F-Dur mit ssixte ajoutée`, übermäßiger Quintsextakkord $f$-a-c-dis, H-Dur, H-Dur-Septakkord usw. Das erste Tonfeld ist sozusagen der Inbegriff von Subdominante/Doppeldominante. Dass aber die Doppeldominante von der Subdominante nur durch eine minimale Differenz getrennt ist, die manchmal die Möglichkeit einer gegenseitigen Vertretung erlaubt, ist auch von den traditionellen Harmonielehren gesehen worden (und in Schenkers Diagrammen erscheint eine Doppeldominante innerhalb einer Kadenz ohnehin als chromatische Variante der IV./II. Stufe).

\section{Zwischen den Zeilen}

Der es-Moll-Akkord in Takt 220 des Kopfsatzes von Mozarts Prager Sinfonie ist satztechnisch eine Kombination aus mehreren Durchgangsnoten. In Schenkers Diagrammen würde er gar nicht als substantieller Zusammenklang erscheinen (sondern als Appoggiatura), und auch die traditionellen Harmonielehren würden den Akkord wahrscheinlich nicht als solchen ernst nehmen.

Simons Theorie versucht, Klänge und ihre harmonischen Wirkungen, die im Lichte der traditionellen Tonalität zwischen den Zeilen stehen, als solche erfahrbar zu machen. Für ihn ist interessant, dass der es-Moll-Akkord - obwohl satztechnisch eindeutig ein Durchgang - für einen kurzen Augenblick einen dunklen Schatten auf das harmonische Geschehen wirft und dass es sich lohnt, den Voraussetzungen dieser Akkordwirkung nachzugehen. Nach Simon könnte die Dunkelheit des Durchgangsakkordes von dem Umstand abhängen, dass einige Takte zuvor (Takt 218) ein G-Dur-Akkord erklang. Die Tatsache der Existenz eines G-Dur-Akkordes lässt den es-Moll-Akkord danach beinahe abgründig erscheinen. G-Dur und es-Moll werden als voneinander abhängig beschrieben, obwohl sie satztechnisch nicht auf derselben Ebene liegen und deswegen nach Schenker gar nicht aufeinander bezogen werden könnten. G-Dur und es-Moll ergänzen sich zu einem Konstrukt. Im Gegensatz zur Äquivalenzwirkung der Funktionen wirken Akkorde, die aus einem Konstrukt herausgeschnitten werden, eher kontrastiv.

\section{Vielseitigkeit der Tonfelder}

Tonfelder können auf allen Ebenen des Tonsatzes wirksam sein. Auch die Aufteilung der Töne eines Tonfelds folgt keinen apriorischen Regeln. So lassen sich die Töne eines Konstrukts beispielsweise in zwei übermäßige Dreiklänge, in einen Dur- und einen Mollakkord, deren Töne in Halbtonschritten voneinander entfernt sind, oder in drei Dur- und/oder Mollakkorde im Großterzabstand aufteilen.

Die Aufteilung kann dabei als unmittelbare Akkordfolge und im satztechnischen Gewand eines Fauxbourdonsatzes erscheinen wie in Mozarts Fantasie KV 475 (Takt 12/13) oder als Anlaufstationen innerhalb eines Formabschnitts wie im Kopfsatz des Klavierkonzerts 


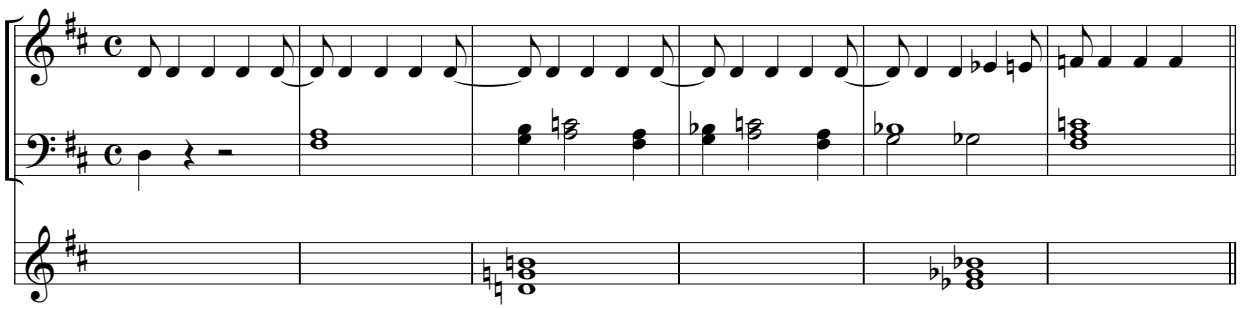

Beispiel 8: W. A. Mozart, Prager Sinfonie, 1. Satz, T. 216-221

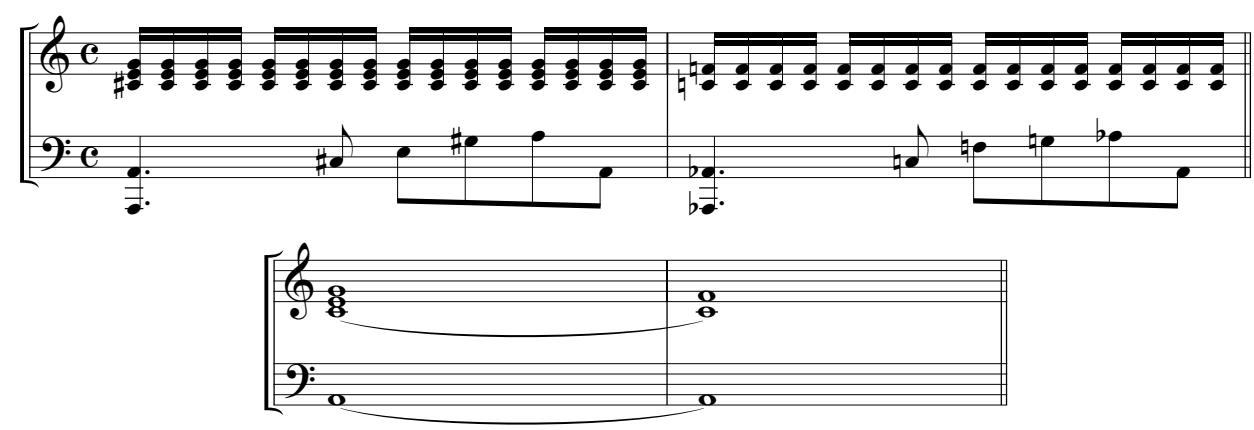

Beispiel 9: W. A. Mozart, Fantasie KV 475, T. 12-13

KV 503 (Takt 324-344: Es-Dur und es-Moll jeweils als lokale Tonika - H-Dur und h-Moll jeweils als lokale Tonika - G-Dur als Halbschlussklang innerhalb der Tonart C-Dur).

Formbildend ist das Konstrukt in der Durchführung von Beethovens Appassionata. Die Durchführung im ganzen führt von einem as-Moll-Akkord zu einem C-Dur-Akkord. Bereits dieses Verhältnis stellt ein Konstrukt dar. Innerhalb des ersten Teils der Durchführung führt der Weg vom erwähnten as-Moll-Akkord zu einem As-Dur-Akkord. Die Stationen des Wegs werden durch eben dasselbe Konstrukt bestimmt: as-E-e-c-As. Nur der C-Dur-Akkord, der den Schluss der Durchührung bildet, ist im ersten Teil weggelassen (vollständig hätte sich die Akkordfolge as - E - e-C - c - As ergeben, in der jeder neue Akkord durch einen
Halbtonschritt vom vorherigen unterschieden ist). Innerhalb des ersten Teils markiert wiederum der e-Moll-Akkord den dunkelsten Punkt (vor allem nach dem friedlichen E-DurAbschnitt zuvor). Mit ihm beginnt der durch Sechzehntel geprägte Abschnitt, an dessen Ende das helle glänzende As-Dur steht. Der dunkelste und der hellste Punkt e-Moll und As-Dur bilden zusammen wiederum ein Konstrukt.

Mehrdeutigkeiten

Differenzierte Theorien stellen in der Regel so viele Möglichkeiten der Deutung zur Verfügung, dass viele Phänomene auf den ersten Blick hin mehrere Interpretationen zulassen. 
Eine Akkordfolge wie g-Moll-H-Dur kann ein Konstrukt bilden oder zu zwei Funktionen gehören (sozusagen T-D bzw. S-T). Die Theorie fordert - gemäß der beschriebenen Logik - dazu auf, die Akkordfolge im konkreten Falle sorgsam auszuhören und unter den vorhandenen Möglichkeiten diejenige auszuwählen, die den Klangcharakter der betreffenden Stelle am besten trifft (und wenn die Akkordfolge Eigenschaften sowohl eines Konstrukts als auch einer Funktionsfolge aufweist, wird man in die Deutung beide Möglichkeiten einbeziehen).

In Schuberts Lied Der Atlas wird man das Tonartenverhältnis g-Moll - H-Dur zwischen den äußeren Abschnitten und dem Mittelteil wegen des Kontrastes als Konstrukt deuten (dasselbe gilt für das Impromptu op. 90,2, in dem die Rahmenteile in Es-Dur einem Mittelteil in h-Moll schroff gegenüberstehen).

Am Ende der Exposition aus der 9. Sinfonie von Bruckner liegt es näher, die Akkorde h-Moll und g-Moll als Ausschnitte aus Funktionen zu deuten. Die Schlusskadenz insgesamt besteht aus den Akkorden: h-Moll - g-Moll e-Moll - C-Dur - b-Moll - F-Dur (auch diese Akkorde sind auf sich selbst und nicht auf eine Tonart bezogen, auch wenn F-Dur den Schlussakkord bildet). Bezieht man die Akkorde auf Funktionen, dann ergibt sich eine einfache Abfolge: Von h-Moll aus steigt Bruckner squintabwärts` zu g-Moll und e-Moll, danach noch einmal um eine `Quinte zu C-Dur. Von da an folgt ein zweifacher 'Quintanstieg`: C-Dur - b-Moll - F-Dur. Somit bildet C-Dur die Mitte, während die benachbarten Akkorde g-Moll, e-Moll und b-Moll ebenso zu einem Tonfeld gehören wie die 'Rand-Akkorde` h-Moll und F-Dur, die Anfang und Schluss der Kadenz bestimmen. Die Tritonuspaare F-Dur/h-Moll und B-Dur/e-Moll bilden nach den Worten von Haas »Vorder- und Rückseite« einer Funktion ab.
Die Pointe bei Bruckner aber steckt im Schlussakkord F-Dur, in den jenes seltsame $h$ hineingemischt wird, über das der Verfasser an einer früheren Stelle einmal geschrieben hat, es bleibe unklar, ob dieser Ton harmonieeigen oder harmoniefremd sei. ${ }^{7}$ Er passt in den Akkord, aber man weiß - vor traditionellem Hintergrund - nicht, auf welche Weise. Nach Simon gehört der Ton $h$ selbstverständlich zu der Funktion, die den F-Dur- und den hMoll-Akkord enthält. Das $h$ ist eine Erinnerung an das äquivalente h-Moll, mit dem die Schlusskadenz begann.

Wer die Akkordfolge h-Moll-g-Moll zu Beginn der Kadenz hört, wird allerdings auch eine leicht abgründige Wirkung beim g-MollAkkord spüren, die von seiner Position nach h-Moll herrührt (man vergleiche, um wie viel weniger drastisch der e-Moll-Akkord nach g-Moll wirkt). Insofern wäre es nicht verfehlt, innerhalb eines harmonischen Rahmens, der auf Funktionen basiert, von einem internen Konstrukt zu sprechen.

Deutlich ist die Synthese aus Funktion und Konstrukt zu Beginn von Regers Introduktion, Passacaglia und Fuge $h$-Moll für zwei Klaviere. Den Rahmen der Eröffnung bildet ein Konstrukt. Bereits die Akkordfolge g-Moll - H-Dur am Ende umfasst sämtliche sechs Töne des Tonfelds. Die Akkorde zu Beginn (h-Moll und der übermäßige Dreiklang $d$-fis-ais) werden ebenfalls aus den Tönen des Konstrukts gebildet.

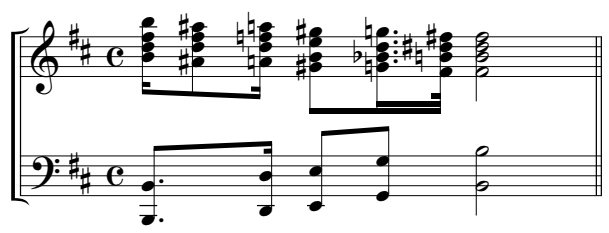

Beispiel 10: Reger, Introduktion, Passacaglia und Fuge $h$-Moll für zwei Klaviere, T. 1 
Andererseits ist auch eine Interpretation durch Funktionen möglich. Fasst man nämlich den übermäßigen Dreiklang als Durchgangsakkord auf (auch dies ist bei Simon zulässig, wenn es dem Klangeindruck entspricht), dann erscheint eine Folge von Funktionen:

- ITonikar: h-Moll/d-Moll

- subdominantes: E-Dur/g-Moll

- ITonikar: H-Dur.

Die Funktionen der Tonika und Subdominante werden jeweils durch zwei äquivalente Akkorde dargestellt. Der Abschluss g-Moll - HDur wirkt so gesehen wie ein Plagalschluss. Die aufreizende Wirkung, welche die Akkordfolge als Konstrukt hätte haben können, wird bei Reger zu der eher harmlosen Wirkung einer Funktionsfolge abgemildert.

Beinahe das Gegenteil findet sich in Schumanns a-Moll-Klavierkonzert (1. Satz, Takt 126). Hier wird die Substanz einer Funktion mit der Wirkung eines Konstrukts angereichert. Der b-Moll-Akkord, der satztechnisch aus Durchgangsbewegungen verschiedener Stimmen resultiert (die Notation der Streicher hebt diesen Aspekt hervor), steht an Stelle eines G-Dur-Septakkordes, der normal, aber auch blasser erschiene: b-Moll und G-Dur gehören zu einer Funktion.

Geht der Blick vom funktionalen Aspekt hin zur kontrastiven Wirkung des Klanges (die durch die Notierung im Klavier suggeriert wird), dann wird man von einem Konstrukt D-Dur/b-Moll sprechen, um damit auszu- drücken, dass der fremde und dunkle b-MollAkkord inmitten von D-Dur- und G-Dur-Akkorden dafür sorgt, dass die vergleichsweise harmlosen Klänge in der Umgebung des bMoll-Akkordes heller und glänzender erscheinen, als man es ohne diesen Akkord erwarten könnte. Die Akkordfolge im Ganzen erhält durch das kurze Konstrukt einen aufreizenden Impuls.

\section{Die Analysen}

Die Lektüre des Buches von Haas ist alles andere als einfach. Die Schwierigkeiten ähneln - wohl nicht zufällig - denjenigen, die bei der Beschäftigung mit den Schriften Schenkers auftauchen. Das hat zum einen mit der erwähnten besonderen Logik der sfunktionalen Analyse $z u$ tun. Zum anderen ist der Sprachstil, den der Autor pflegt, äußerst lakonisch. Das wiegt um so schwerer, als es mit knappen und nüchternen Sätzen kaum möglich ist, einen Leser an die ästhetische، Seite der Theorie heranzuführen, daran also, dass die begriffliche Seite der Theorie detailliert und konkret auf Klangwirkungen am Kunstwerk zielt. Mit der Verlinkung von Begriff und Wahrnehmung erfüllt die Theorie aber überhaupt erst ihren Sinn. Ein hartes Brot sind des weiteren die Analysen. Reichtum und Genauigkeit der Beobachtungen in den Diagrammen stehen auch hier in schroffem Kontrast zur geringen Mittei-

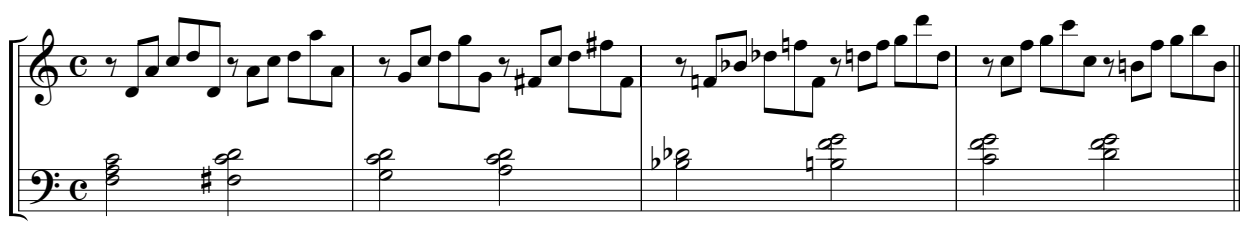

Beispiel 11: Robert Schumann: Klavierkonzert a-Moll op. 54, 1. Satz, T. 124-127 
lungsfreudigkeit des Autors im Text. Der Rezensent gesteht, dass sich ihm in einigen Analysen bislang noch nicht alle Details erschlossen haben. Aber immerhin darf gesagt werden: Manches, was auf den ersten Blick willkürlich wirkt, kann sich nach längerer Beschäftigung als sehr triftig erweisen. So schien etwa die Verteilung des 'Konstrukts IIb in Liszts Klavierstück Am Grabe Richard Wagners auf die Takte 2, 4, 6 und 39 kaum nachvollziehbar. Man muss allerdings den Details des Tonsatzes aufmerksam folgen und alle (wirklich alle!) Hinweise von Haas berücksichtigen, um festzustellen, dass die Interpretation fundiert ist.
Sorgfalt und Ausdauer beim Nachvollzug sowie auch ein Wille dazu, das im Diagramm Angebotene in ein Hörerlebnis zu übersetzen, sind bei Haas zwingende Voraussetzungen zum Verständnis der Analysen. Solche Anforderungen sind in der musiktheoretischen Literatur sicherlich unpopulär - aber wenn man einem Buch Unpopularität bescheinigt, kann das auch ein Kompliment sein.

Michael Polth

\section{Anmerkungen}

1 Lendvai 1971.

2 Gardonyi/Nordhoff 2002.

3 Taruskin 1996.

4 In einer seiner jüngsten Veröffentlichungen (2004) bildet eine durch das erste Notenbeispiel wiedergegebene Dreiklangsfolge (E-Dur-c-Moll), welche in der Theorie

\section{Literatur}

Cohn, Richard (2004), „Uncanny Resemblances: Tonal Signification in the Freudian Age", Journal of the American Musicological Society 57/2, 285-323.

Gardonyi, Zsolt / Hubert Nordhoff (2002), Harmonik, 2. Aufl., Wolfenbüttel: Möseler. Haas, Bruno (2003), Die freie Kunst. Beiträge zu Hegels Wissenschaft der Logik, der Kunst und des Religiösen, Berlin: Duncker \& Humbolt.

Lendvai, Ernö (1971), Béla Bartok. An Analysis of His Music, London: Kahn.

Taruskin, Richard (1996), Stravinsky and the Russian Traditions. A Biography of the Works Through Mavra, London: Oxford University Press.

Polth, Michael (2001), »Nicht System - nicht Resultat. Zur Bestimmung von harmonischer Tonalität», Musik \& Ästhetik 18, 12-36. 